\title{
Paradiso Terrestre su pergamena: il canto XXVIII del Purgatorio disegnato da Sandro Botticelli
}

\author{
Dagmar Korbacher \\ Kupferstichkabinett Staatliche Museen zu Berlin \\ d.korbacher@smb.spk-berlin.de
}

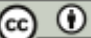

\section{Riassunto}

Questa "lectura Dantis" parte da uno dei celebri disegni di Sandro Botticelli per la Commedia, conservati presso il Kupferstichkabinett di Berlino, cioè dal disegno che l'artista fece per il canto ventottesimo del Purgatorio intorno al I488-I492 circa. Questo canto offre una varietà di motivi che sottolineano in modo esemplare i rapporti tra la concezione poetica di Dante e quella artistica di Botticelli. Il profondo interesse di Botticelli per Dante, e più precisamente per il Paradiso Terrestre descritto dal poeta, emerge in modo evidente anche nella celebre Primavera, un dipinto che rappresenta l'ambiente del Paradiso Terrestre dantesco in modo esemplare.

Parole chiave: Disegno; Paradiso Terrestre; Sandro Botticelli; Primavera; Matelda; Beatrice; Virgilio; Stazio; Venere; Madonna.

\begin{abstract}
This “lectura Dantis” starts from one of Sandro Botticelli's famous drawings for Comedy, preserved at the Kupferstichkabinett in Berlin, i.e. from the drawing that the artist made for the twenty-eighth canto of Purgatory around I488-I492 (or so). This canto offers a variety of reasons that exemplarily underscore the relations between Dante's poetic conception and Botticelli's artistic one. Botticelli's deep interest for Dante, and more precisely for the Eden described by the poet, clearly emerges also in the famous Spring, a painting that represents the ambience of Dante's Eden in an exemplary way.
\end{abstract}

Keywords: Drawing; Eden; Sandro Botticelli; Spring; Matelda; Beatrice; Virgilio; Statius; Venere; Madonna. 


\section{Una “lectura Dantis botticelliana”}

$\mathrm{D}$ ante Alighieri e Sandro Botticelli ${ }^{1}$ sono due nomi che come pochi altri rappresentano l'Italia, la cultura italiana, Firenze e la cultura rinascimentale. I due ovviamente non vissero nella stessa epoca - il grande pittore nacque infatti quasi due secoli dopo il grande poeta - eppure trovano un punto d'incontro laddove la parola, in quanto idea, diventa immagine, ossia nell'arte del disegno. I celebri disegni di Sandro Botticelli per la Commedia dantesca sono una testimonianza straordinaria ed unica di come Botticelli abbia letto e interpretato Dante. Tali disegni, per la loro singolarità e per il tentativo di illustrare l'intero poema, possono essere intesi come un vero commento visivo della Commedia, nato da un'attenta riflessione del pittore sull'opera di Alighieri. Che l'artista si interessasse di Dante era ben noto ai suoi primi biografi. Giorgio Vasari racconta infatti che Botticelli, dopo aver terminato gli affreschi nella Cappella Sistina a Roma, “...se ne tornò subitamente a Fiorenza. Dove per essere persona sofistica comentò una parte di Dante, e figurò lo Inferno e lo mise in stampa, dietro al quale consumò di molto tempo; per il che non lavorando, fu cagione di infiniti disordini alla vita sua" (Vasari, Le Vite - Vita di Sandro Botticelli: 516-517). Tuttavia Vasari apparentemente conosceva soltanto delle stampe ed illustrazioni della prima parte della Commedia che associava all'artista, cioè probabilmente le incisioni che accompagnano l'edizione commentata di Cristoforo Landino del I48I (cfr. Keller 2000, Dreyer 1984); d'altra parte il cosiddetto Anonimo Magliabechiano (possibilmente identico con Vincenzo Borghini; cfr. Stapleford I995: 397), nel 1540 circa, scriveva nelle sue Vite di artisti che Botticelli "Dipinse et storiò un Dante in carta pecora a Lorenzo di Piero Francesco de' Medici che fu cosa maravigliosa tenuto" (Firenze, Biblioteca Nazionale di Firenze, Cod. Magliabechiano, XVII, I7, fol. 84; cit. da Stapleford 1995: 408). Tale osservazione non solo ricorda l'impegno di Botticelli come illustratore, ma offre anche ulteriori informazioni sulla qualità dell'opera e sul supporto per-

I. Questo testo nasce da una conferenza tenuta presso l'università di Potsdam il I7 giugno $20 \mathrm{I} 4$, in occasione delle giornate dedicate alle Lecturae Dantis; desidero ringraziare per l'invito la prof.ssa Cornelia Klettke, che condivide la passione per i disegni danteschi di Botticelli, il prof. Rossend Arqués per avermi concesso di pubblicare sulla sua rivista questo contributo e la dott.ssa Beatrice Alai per la revisione del testo italiano.Il celebre ciclo dei disegni di Botticelli per la Commedia comprendeva originariamente centodue fogli (uno per ogni canto più una visione generale dell'Inferno e un'immagine che si estende su due fogli. Di questi ne restano novantadue: ottantacinque fogli sono conservati presso il Kupferstichkabinett di Berlino (Cod. Ham. 2oI), mentre altri sette si trovano nella Biblioteca Apostolica Vaticana (Cod. Reg. lat. I846). Vista la vastità della bibliografia relativa ai disegni, mi limito in questa sede a segnalare alcuni riferimenti principali: Risset (2008); Schulze Altcappenberg (2000a); Schulze Altcappenberg (2000b); Bellini (1990); Clark (1976); Dombrowski (I996); Gentile (2000); Girolami Cheney (I988); Lightbown (I978); Batard (I952); Toesca (I922); Venturi (I92I); Lippmann (I9212); Lippmann (I883). 
gamenaceo, oltre che sul committente dell'artista, ovvero Lorenzo di Pierfrancesco de' Medici. Come è noto, egli fu committente e proprietario di alcuni dei dipinti più famosi di Botticelli, cioè della Primavera, della Pallade con il Centauro e della Nascita di Venere, oggi conservati nella Galleria degli Uffizi a Firenze. Lorenzo di Pierfrancesco, amante delle lettere, verosimilmente condivise e favorì il grande interesse di Botticelli per Dante. Inoltre, è probabile che questa "cosa maravigliosa", "un Dante in cartapecora", sia sopravvissuto e che si tratti del ciclo quasi completo di disegni della Divina Commedia conservati in parte presso il Kupferstichkabinett di Berlino ed in parte presso la Biblioteca Apostolica Vaticana.

Tuttavia, tali disegni non possono essere considerati come commento visivo alla base di una lectura Dantis botticelliana senza accennare a due problemi correlati: la prima osservazione riguarda l'opinione di Vasari, secondo cui Botticelli sarebbe stato un artista illetterato; la seconda verte invece sulla diffusa convinzione che la visione dell'artista sarebbe stata influenzata dal commento di Cristoforo Landino sulla Commedia, questione che si contrappone ed al contempo si lega strettamente a quanto affermato da Vasari. L'opinione dispregiativa di Vasari sull'ignoranza di Botticelli che "senza avere lettere o apena saper leggere comenta Dante" (Vasari, Le Vite - Vita di Sandro Botticelli: 519) si è tramandata per secoli, più o meno invariata, ed è stata confutata solo negli ultimi decenni. Botticelli oggi passa piuttosto per pictor doctus (cfr. Dombrowski 1996: 64). Possiamo ipotizzare che Botticelli avesse familiarità con i colti personaggi della cerchia di Lorenzo il Magnifico, ed appunto con Cristoforo Landino, come pure con il suo probabile committente Lorenzo di Pierfrancesco de' Medici. Infatti, non sono da sottovalutare la cultura e l'erudizione dello stesso Botticelli, grazie a cui egli si cimenta in straordinario commento figurativo (cfr. ibid.), dimostrando di saper interpretare in modo personale e raffinato il testo della Commedia. Botticelli non si interessa tanto al sensus allegoricus ma si attiene piuttosto al sensus litteralis, illustrando innanzitutto le tappe del viaggio di Dante nell'aldilà (cfr. Schulze Altcappenberg 20oob: 34 ). In questo l'artista è molto vicino alle indicazioni del poeta e rappresenta in modo puntuale le informazioni contenute nel testo. Botticelli sfrutta l'efficacia visiva del sensus litteralis e raffigura gli spazi della Commedia come luoghi concreti anziché come idee neoplatoniche (cfr. Dombrowski 1996: 64), illustrando le scene sia in maniera analitica che compendiaria. Anche nell'impostazione scenica generale Botticelli si appoggia fedelmente alla concezione dantesca e realizza la struttura dei disegni mantenendo uno stretto legame col testo, cercando di rispecchiare non solo il significato delle parole ma, come vedremo più avanti, anche lo stile usato dal poeta (cfr. Fallani 197I: IOI). L'abilità dell'artista sta infatti nel rappresentare i singoli episodi servendosi di un complesso sistema narrativo in cui i paesaggi 
delle tre cantiche dantesche fanno da sfondo agli incontri del poeta con i vari personaggi, ad esempio nell'episodio di Matelda. Giustamente, la critica ha messo in luce come "in questo ciclo [...] uno dei massimi maestri dell'epoca misura la sua arte con una delle opere della letteratura mondiale più ricche di immagini, ma anche più difficili per la complessità delle astrazioni, la pienezza dei motivi e dei riferimenti" (Schulze Altcappenberg 200ob: 16).

\section{Il Canto XXVIII del Purgatorio disegnato da Botticelli}

Come esempio di lectura Dantis che illustri il punto di vista di Botticelli, abbiamo scelto il disegno che l'artista fece per il canto ventottesimo del Purgatorio intorno al I488-I492 circa (cfr. Schulze Altcappenberg 2000a: I98; Venturi 192I: 99-IO0) [fig. I]. Come la maggior parte dei disegni del ciclo dantesco, esso consiste in uno schizzo a punta metallica dal segno delicato e quasi impercettibile, ripassato in parte con l'inchiostro marrone (cfr. Oltrogge/Fuchs/Hahn 2000). Questo canto offre un'ampia gamma di motivi che sottolineano in modo esemplare i rapporti tra la concezione poetica di Dante e quella artistica di Botticelli.

Sia nei canti che nei disegni che precedono il canto Pg. XXVIII il lettore ovvero lo spettatore si avvicina man mano al Paradiso Terrestre: per facilitare questo percorso, Botticelli inserisce nelle illustrazioni degli episodi alcuni riferimenti topografici che aiutano l'osservatore ad orientarsi nei vari canti del poema. Così lo spettatore, che giunge con Dante e Virgilio all'isola dove il monte del Purgatorio si erge imponente sull'oceano, può usufruire di una panoramica dell'intero Purgatorio accompagnata dalla raffigurazione degli avvenimenti del primo canto; anche il Paradiso Terrestre è ben visibile in cima alla montagna [fig. 2]. Questa veduta generale mostra dunque a grandi linee gli avvenimenti delle tappe successive: infatti, in tutti i disegni si intuisce chiaramente la volontà di ottenere una vera continuità narrativa, in quanto l'artista, in ciascun disegno, non solo cerca di visualizzare tutti gli eventi di un unico canto ma spesso inserisce anche riferimenti a quello precedente e a quello successivo. Sebbene tali riferimenti non siano presenti nel testo di Dante, essi divengono una sorta di "sovra scenario" reiterato che aiuta la memoria del lettore-spettatore a non perdere il filo degli avvenimenti durante l'incedere degli episodi, che ogni volta introducono nuovi elementi. Botticelli riesce così a mantenere, nonostante la specificità dei singoli canti, una visione d'insieme che allude alla destinazione ultima del viaggio dantesco, ovvero il Paradiso: tale ascesa viene ricordata anche attraverso la ripetizione dei personaggi, che con i gesti delle mani indicano allo spettatore la direzione del percorso, mentre nell'Inferno e nel Purgatorio è rivolta verso alto (cfr. Dombrowski 1996: 50-5I). Che l'artista scelga di ricreare la "continuità narrativa" è 
ben evidente anche nel disegno del canto ventottesimo: nonostante l'episodio si svolga interamente nel Paradiso Terrestre, sono ancora visibili, in basso a sinistra, le pendici rocciose della montagna che Dante ha superato fino a quel momento [fig. I]. Al contrario, nel disegno che illustra il canto precedente $(P g$. XXVII) [fig. 3], lo spettatore intravede già nel margine superiore della scena gli alberi della foresta del Paradiso Terrestre, e la memoria di tale luogo persiste nel disegno per il primo canto del Paradiso [fig. 4]: in quest'ultimo è presente di nuovo il paesaggio con gli alberi del Paradiso Terrestre già raffigurato nel disegno del canto XXVIII del Purgatorio, dove a sua volta si intuisce la struttura circolare del Paradiso grazie ai semicerchi dipinti nel margine superiore del foglio [fig. I]. Tale linguaggio espressivo, basato sulla concatenazione visuale, si può interpretare come il corrispettivo dello stile letterario di Dante, che utilizza versi in terza rima (cfr. Dombrowski 1996: 49).

Abbiamo messo in luce come la presenza di uno scenario di fondo, ripetuto lungo i vari canti, serva da collegamento tra tutti i disegni che, presi singolarmente, non perdono mai l'aspirazione a rappresentare analiticamente tutti gli avvenimenti del canto corrispondente.

Lartista sovrappone al medesimo "fondale" un primo piano mutevole, rappresentando gli stessi personaggi più volte ma cambiandone la posizione $\mathrm{e}$ la dimensione, in modo tale da far percepire all'osservatore l'evoluzione spazio-temporale della rappresentazione. Tali figure assumono il ruolo di guida per l'occhio dello spettatore, permettendogli di procedere lungo il cammino assieme a loro (cfr. Schulze Altcappenberg 200ob: 3I-32).

Tornando al canto ventottesimo del Purgatorio, Dante e i suoi compagni sono arrivati in cima alla montagna. La prima rappresentazione del gruppo dei tre viaggiatori, vicino al margine sinistro del disegno, mostra il loro arrivo alla foresta del Paradiso Terrestre [figg. I e 5]. Virgilio appare assorto nei suoi pensieri, e resta immobile. Il suo atteggiamento rivela una timidezza ma anche una certa malinconia. La sua posizione è leggermente arretrata rispetto a quella di Dante, dal momento che, come egli stesso ha spiegato alla fine del canto precedente, il suo compito di guida è ormai compiuto e di lì a poco lascerà Dante per tornare al Nobil Castello (cfr. Pg. XXVII, I39: "Non aspettar mio dir più né mio cenno"). Il primo dei tre pellegrini, Stazio, volge lo sguardo verso l'alto, portandosi una mano alla fronte. Similmente Dante, collocato alle sue spalle, si ripara il volto con una mano. Di primo acchito il gesto di Stazio e di Dante potrebbe essere frainteso: sembrerebbe infatti che i due personaggi si vogliano proteggere da una luce abbagliante, che impedisce loro di vedere bene; a suffragio di tale interpretazione si può utilizzare ciò che nel canto precedente Virgilio dice a Dante: "Vedi lo sol che 'n fronte ti riluce" (Pg. XXVII, I33). Nel nostro caso però Dante guarda nella direzione opposta rispetto alla quella in cui si volgeva nel disegno del canto precedente, 
dove appunto Virgilio commentava le parole poc'anzi ricordate; diversamente, all'entrata del Paradiso Terrestre non c'è più bisogno di proteggersi gli occhi dai raggi del sole, poiché le chiome degli alberi smorzano la luce accecante del primo mattino: "la divina foresta spessa e viva, ch'a li occhi temperava il novo giorno" (Pg. XXVIII, 2-3).

Il concetto di "divina foresta e spessa e viva" era pensato da Botticelli come una ricca vegetazione ombrosa, come indicano i fasci di linee fitte che riempiono le chiome degli alberi ed i numerosi tratteggi a punta metallica che suggeriscono la presenza di un bosco "spesso", cioè folto e intricato. Ecco che allora il gesto di Stazio va inteso piuttosto come un tentativo di guardare con attenzione, con un'intensità tale che lascia trapelare il desiderio di osservare attorno a sé, secondo quanto descritto all'inizio del il canto: "Vago già di cercar dentro e dintorno" (Pg. XXVIII, I).

Il gesto di Dante esprime stupore, e sembra che egli voglia afferrare con la mano il lieve vento che gli accarezza la fronte e muove le chiome degli alberi, disegnati con linee lunghe e fluide: "Un'aura dolce, sanza mutamento / avere in sé, mi feria per la fronte / non di più colpo che soave vento" (Pg. XXVIII, 7-9). Il tentativo di intravedere la brezza tra le fronde spiega anche il gesto del poeta di guardare verso l'alto.

L'arrivo del terzetto nel Paradiso Terrestre è disegnato su una parte di pergamena che è stata aggiunta. Non conosciamo la ragione di questa addizione, tuttavia è evidente che venne fatta dall'artista prima che venisse scritto il testo (del canto precedente, cioè Pg. XXVII) sul verso del foglio. Si può ipotizzare che Botticelli avesse incontrato particolari difficoltà nella rappresentazione di questo episodio, come testimoniano i diversi pentimenti, e che i numerosi rifacimenti avessero causato danni irreversibili alla superficie della pergamena, rendendo necessaria l'aggiunta di un nuovo pezzo.

Il canto prosegue con la descrizione delle chiome degli alberi che si piegano verso ovest, nella direzione in cui si proiettano le ombre del primo sole mattutino: "per cui le fronde, tremolando, pronte / tutte quante piegavano a la parte / u' la prim' ombra gitta il santo monte" (Pg. XXVIII, IO-I2). Analogamente nel disegno di Botticelli le chiome degli alberi si piegano verso destra, nella direzione in cui si muovono i personaggi: tale direzione corrisponde, come ci informa Dante, alla traiettoria su cui cadono le ombre, cioè verso ovest. Da ciò si deduce che il sole sta alle spalle dei viaggiatori: di conseguenza si può escludere che i personaggi si stiano parando gli occhi dalla luce del sole. Un'ulteriore prova che Stazio e Dante non si stiano proteggendo la vista dal sole è implicitamente fornita già dal disegno precedente, che corrisponde al canto XXVII del Purgatorio: alla fine del canto, il sole appena sorto splende sul volto di Dante, ma lui si volge nella direzione opposta [fig. 3]. 
Il canto XXVIII prosegue quindi con una descrizione dettagliata della foresta del Paradiso Terrestre. Essa mette in luce i suoni e le melodie della natura: "[...] li augelletti per le cime / lasciasser d'operare ogne lor arte; ma con piena letizia l'ore prime, / cantando, ricevieno intra le foglie, / che tenevan bordone a le sue rime, / tal qual di ramo in ramo si raccoglie / per la pineta in su 'l lito di Chiassi, / quand'Eolo scilocco fuor discioglie" (Pg. XXVIII, I4-2I). Lartista qui non rappresenta i cinguettii, la musica, i rumori ed i suoni della foresta, rinuncia pure alla raffigurazione degli uccelli, verosimilmente nascosti tra il fogliame, poiché conscio di non poter render giustizia, col disegno, al loro canto, vero pregio che li distingue ben più del piumaggio sontuoso. Di conseguenza, Botticelli rinuncia completamente alla rappresentazione degli elementi acustici; tuttavia, possiamo avvertire nel tratteggio - soprattutto nelle piccole linee di tratteggio che riempiono la chioma dell'albero sulla sinistra - un rimando al fruscio del fogliame, descritto dal poeta con dovizia di particolari e messo in rilievo per onomatopea grazie all'allitterazione della lettera "r": "di ramo in ramo si raccoglie" (Pg. XXVIII, I9).

L'incedere di Dante lungo il percorso nel Paradiso Terrestre è descritto come "lento lento" (Pg. XXVIII, 5) ed è caratterizzato da "lenti passi" ( $P g$. XXVIII, 22). Questo procedere solenne è messo in risalto anche dalle vesti del poeta che cadono in pieghe a canne d'organo, in una tensione lineare che allude ad un movimento molto contenuto. Le terzine successive narrano dell'avanzare di Dante nel fitto bosco: "Già m’avean trasportato i lenti passi / dentro a la selva antica tanto, ch'io / non potea rivedere ond'io mi 'ntrassi" (Pg. XXVIII, 22-24). La raffigurazione di Botticelli si sposta progressivamente a destra, raggiungendo il centro del foglio: qui vediamo che i tre pellegrini, in cammino attraverso la selva del Paradiso Terrestre, giungono nei pressi di un piccolo fiume [fig. 6]. Sotto ai loro piedi si stende un tappeto erboso, disegnato con tocchi brevi ed intensi, dal profilo ricurvo, in punta metallica. Allo stesso modo Dante si sofferma a commentare l'acqua del fiume, che piega le erbe che crescono sulla riva: "con sue picciole onde / piegava l'erba che'n sua ripa uscìo" (Pg. XXVIII, 26-27).

Ma è soprattutto nella rappresentazione del contrasto tra la trasparenza dell'acqua e l'oscurità della foresta ombrosa che l'arte di Botticelli raggiunge i suoi limiti: "Tutte l'acque che son di qua più monde, / parrieno avere in sé mistura alcuna, / verso di quella, che nulla nasconde, / avvegna che si mova bruna bruna / sotto l'ombra perpetua, che mai / non lascia sole ivi né luna" (Pg. XXVIII, 28-33). Il recto della pergamena, che corrisponde qui al lato del carniccio ed è quindi più bianco e levigato del verso (il lato del pelo), è perfettamente congeniale alla raffigurazione della purezza dell'acqua. Manca tuttavia un netto contrasto con l'oscurità della selva, nonostante tra gli alberi vi sia un reticolo di linee tratteggiate rapidamente, che dà l'impressione 
di una certa ombreggiatura. È ipotizzabile che fosse prevista una coloritura successiva del disegno, che avrebbe donato in modo evidente un'aria "bruna bruna" alla foresta ed un aspetto ombroso al luogo: del resto, l'esistenza di alcuni fogli colorati appartenenti alla Commedia botticelliana costituiscono una prova a favore di un progetto iniziale, mai portato a termine, di realizzare un'opera completa a colori (cfr. Oltrogge/Fuchs/Hahn 2000: 338-34I).

Nella parte destra del disegno, il paesaggio è dominato da un corso d'acqua in forma di ampio arco, che isola una zona circolare dal resto della foresta e impedisce ai poeti di continuare il cammino. Dante descrive come, nonostante questo ostacolo costringa i suoi piedi a fermarsi, i suoi occhi vadano oltre, attraversino il fiume e penetrino la foresta verdeggiante: "Coi piè ristretti e con li occhi passai / di là dal fiumicello, per mirare / la gran variazion d'i freschi mai" (Pg. XXVIII, 34-36). Lì il poeta vede una donna sola, che mentre canta raccoglie dei fiori: "una donna soletta che si gia / e cantando e scegliendo fior da fiore / ond'era pinta tutta la sua via" (Pg. XXVIII, 40-42). Dante, contrariato dall'impedimento del fiume, si rivolge alla donna con queste parole: 'Deh, bella donna, che a' raggi d'amore / ti scaldi, s'i' vo' credere a' sembianti / che soglion esser testimon del core, / vegnati in voglia di trarreti avanti", / [...] "verso questa rivera, / tanto ch'io possa intender che tu canti" (Pg. XXVIII, 43-48). La donna esaudisce così la sua preghiera, poiché si gira e si incammina verso di lui; essa si sposta quindi da destra a sinistra, procedendo in direzione opposta rispetto ai tre poeti. Così la scena viene a concentrarsi nel centro del foglio grazie al movimento di Dante e dei suoi compagni da sinistra a destra ed a quello della donna che, raccogliendo i fiori, va da destra a sinistra. La donna, della quale Dante non sa ancora il nome, ovvero Matelda, assomiglia a una ninfa. L'incontro tra questi personaggi costituisce il motivo centrale del canto: il dialogo tra Matelda e Dante, la donna spiega al poeta il Paradiso Terrestre.

Nella rappresentazione di Botticelli il movimento danzante di Matelda, che mette i piedi uno avanti all'altro come se stesse ballando - "Come si volge, con le piante strette / a terra e intra sé, donna che balli, / e piede innanzi piede a pena mette" ( $P g$. XXVIII, 52-54) - pervade l'intero corpo della donna, coinvolgendo anche le vesti: si tratta di un motivo tipicamente botticelliano che Aby Warburg definì bewegtes Beiwerk (Warburg I893: 4) e che Pietro Toesca individuò come il nucleo di quello che per noi ancora costituisce l'arte di Botticelli: "Fu il Botticelli [...] a segnare così la donna che sceglie fior da fiore, tanto è la grazia dell'atto e l'agile moto nei pochi tratti leggeri? Poi ella si colge ridendo, accennando al Poeta con uno di quei gesti alti e ritmici che d'ora innanzi nei disegni parranno moderare musicalmente il moto fluttuante delle persone e delle vesti” (Toesca 1922: 17). Nel disegno il ritmo danzante della donna appare in contrasto con il movimento "lento 
lento" dei passi di Dante e dei suoi compagni e si manifesta in particolar modo nella veste fluttuante di Matelda, così diversa dalle vesti dei poeti che ricadono in rigide pieghe. Di certo una coloritura del disegno avrebbe reso miglior giustizia anche a "i vermigli e in su i gialli / fioretti ..." (Pg. XXVIII, 55-56), donando loro una tinta rossa e gialla in accordo col testo dantesco. D'altra parte la scena ritrae fedelmente la posizione delle figure come descritte dal poema, come si osserva per il personaggio di Matelda che, secondo i versi, è colta nell'atto di volgersi verso il poeta ("volsesi ... verso me", Pg. XXVIII, 55-56). Inizialmente la donna è rappresentata sulla destra della scena, intenta a cantare ed a raccogliere fiori, mentre in un secondo momento essa si volge verso Dante che le parla, soddisfacendo così il desiderio del poeta: "e fece i prieghi miei esser contenti, / sì appressando sé, che 'l dolce suono / veniva a me co' suoi intendimenti" (Pg. XXVIII, 58-60). Matelda incontra Dante sulla riva del fiume, dove l'erba è bagnata dalle onde: "Tosto che fu là dove l'erbe sono / bagnate già da l'onde del bel fiume" (Pg. XXVIII, 6I-62). Mentre Dante narra di come Matelda gli abbia fatto dono del suo sguardo ("di levar li occhi suoi mi fece dono" - Pg. XXVIII, 63), Botticelli raffigura il momento precedente, in cui la donna tiene gli occhi abbassati: in tal modo, egli rinuncia a rappresentare sia la bellezza degli occhi della donna, paragonabili a quelli di Venere, sia il suo sorriso. La mancanza di scambi di sguardi distingue questo disegno da quelli del Paradiso: in essi gli sguardi vengono rappresentati, divenendo quasi una cifra stilistica dell'artista, ed al contempo si caricano di significato evocativo (“bedeutsames Blickgeschehen": cfr. Klettke 20I0: 70).

Proseguendo la lettura del testo di Dante, si incontra una descrizione puntuale che riguarda l'ampiezza del fiume che separa Dante e Matelda: "Tre passi ci facea il fiume lontani” ( $P g$. XXVIII, 70). Tre passi è la distanza che corrisponde più o meno anche a quella che separa i due protagonisti disegnati da Botticelli. Mentre Dante si serve dell'aneddoto di Serse e dell'Ellesponto per spiegare l'invalicabilità dell'abisso (cfr. Pg. XXVIII, 7I-75), Botticelli disegna un alto tronco tra la donna e il poeta per sottolineare la separazione fisica dei due personaggi, il cui dialogo è messo in evidenza dalla gestualità caricata. Matelda risponde alla domanda di Dante, che per primo le ha rivolto la parola: "E tu che se' dinanzi e mi pregasti, / dì s'altro vuoli udir; ch'i' venni presta / ad ogne tua question tanto che basti" (Pg. XXVIII, 82-84). Fedele a questa indicazione Botticelli raffigura Dante un po' in avanti rispetto agli altri due poeti.

Qui comincia il dialogo vero e proprio tra Matelda e Dante che occupa quasi tutto il resto del canto ( $P g$. XXVIII, 76-I44): i due personaggi rimangono gli unici protagonisti della scena, e parlano del luogo in cui si trovano. Si tratta indubbiamente di una sfida per il disegnatore, che qui dà prova di straordinaria capacità di sintesi visuale (cfr. Dombrowski 1992: 47). Inoltre, 
il dialogo tra Matelda e Dante anticipa i discorsi tra Dante e Beatrice nel Paradiso. A questo punto conviene chiedersi quali elementi del dialogo tra il poeta e Matelda si possano rintracciare nel disegno. Infatti, osservando la postura delle teste, dei corpi, ed i gesti si capisce il tema della conversazione: la mano destra di Dante è sollevata in un rispettoso gesto di saluto, indicando al contempo la brezza leggera che attira l'attenzione del poeta; con la sinistra egli invece indica il fiume, come per domandare il significato dell'acqua. $\mathrm{Si}$ capisce perciò che il soggetto della conversazione sono i fenomeni naturali che suscitano la curiosità di Dante, cioè l’acqua trasparente ed i suoni della foresta provocati dalle correnti d'aria: "L'acqua', diss'io, 'e 'l suon de la foresta / impugnan dentro a me novella fede" (Pg. XXVIII, 85-86). Le mani di Matelda e quelle di Dante formano una posizione chiastica. Anche Matelda solleva la mano destra, come per spiegare al poeta il movimento dell'aria, mentre il palmo della sinistra è rivolto verso Dante, in un gesto che sembra voler frenare le domande incalzanti del poeta, a cui essa si appresta a rispondere. La destra, puntata verso l'alto, suggerisce a Dante quale sia l'origine dei fenomeni che lo turbano, ovvero il "Sommo Ben": "Lo sommo Ben, che solo esso a sé piace, / fé l'uom buono e a bene, e questo loco / diede per arr'a lui d'etterna pace" (Pg. XXVIII, 9I-93). I versi che seguono ("Per sua difalta qui dimorò poco ... e libero" - Pg. XXVIII, 94-IO2) riguardano la natura del luogo e la natura dell'uomo ed il legame insolubile che esiste tra i due, che tuttavia non sono rappresentati nel disegno di Botticelli. L'artista però riprende il motivo del cerchio, che caratterizza i versi IO3-IO5: "Or perché in circuito tutto quanto / l'aere si volge con la prima volta, / se non li è rotto il cerchio d'alcun canto ..." (Pg. XXVIII, I03-IO5). È proprio il simbolo del cerchio che aiuta Botticelli a raffigurare il passaggio tra il Purgatorio ed il Paradiso. Nell'Inferno e nel Purgatorio gli spazi sono circolari. Anche nel disegno del canto ventottesimo del Purgatorio raffigurante il Paradiso Terrestre il cerchio è un indicazione spaziale, mentre subito dopo, nel Paradiso diventa una visualizzazione astratta, geometrica, una superficie piana piuttosto che uno spazio (cfr. Dombrowski I992: III; Dombrowski 1996: 59-60). Di conseguenza, nel disegno del secondo canto del Paradiso, quest'ultimo è raffigurato da una struttura schematica fatta di sfere concentriche [fig. 7]. La trasformazione del motivo circolare da uno spazio reale ad uno astratto giunge alle estreme conseguenze con la scomparsa totale dell'indicazione dello spazio, quando nei disegni finali per il Paradiso l'artista non definisce più lo spazio delle scene, ma sfrutta la non finitezza della pergamena come segno dell'infinito (cfr. Klettke 20IO: 7I). Nelle immagini del Paradiso terrestre le linee curve, disegnate in secondo piano in prossimità del margine superiore dei fogli, danno l'impressione del dilatarsi dello spazio, allargandosi a cerchi concentrici: sembrerebbe 
che Botticelli avesse voluto misurare l'estensione del giardino dell'Eden servendosi dei tratti briosi della punta metallica [figg. I e 6].

Anche l'analisi della rappresentazione dei fiumi del Paradiso aiuta a comprendere meglio una simile concezione spaziale, dal momento che anch' essi sono caratterizzati da un andamento curvilineo. Nel disegno del canto XXVIII del Purgatorio le acque del fiume separano Matelda dal gruppo maschile, racchiudendola in una radura che assume quasi il valore di Hortus Conclusus (cfr. Bashir-Hecht 1985: I033); Botticelli si serve di questo escamotage anche per alludere a un confronto tra la figura di Matelda e quella della Madonna. Inoltre, la forma circolare dei fiumi funge per dimostrare che essi, diversamente dai fiumi terrestri, non nascono da una sorgente: "Lacqua che vedi non surge di vena / che ristori vapor che gel converta, / come fiume ch'acquista e perde lena; / ma esce di fontana salda e certa, / che tanto dal voler di Dio riprende, / quant'ella versa da due parti aperta" (Pg. XXVIII, I2I-I26). Anche la bipartizione del fiume allude ad un concetto preciso: le due braccia di fiume - una davanti ed una dietro, oppure dal punto di vista di Matelda una a destra ed una a sinistra rappresentano il Lete e l'Eunoe: "Da questa parte con virtù discende / che toglie altrui memoria del peccato; / da l'altra d'ogne ben fatto la rende. / Quinci Letè; così da l'altro lato / Eunoè si chiama ..." (Pg. XXVIII, I27-I3I).

Le spiegazioni di Matelda terminano con un riferimento ai due accompagnatori di Dante, i poeti antichi Virgilio e Stazio, cioè "Quelli ch'anticamente poetaro/ l'età de l'oro e suo stato felice, / forse in Parnaso esto loco sognaro" (Pg. XXVIII, I39-I4I). Nel finale del canto, Dante si volge brevemente a guardare i compagni, accennando una riverenza, per poi tornare a fissare Matelda: "Io mi rivolsi 'n dietro allora tutto / a' miei poeti, e vidi che con riso / udito avean l'ultimo costrutto; / poi a la bella donna torna' il viso" (Pg. XXVIII, I45-I48). Lo sguardo dello spettatore (ma non quello di Dante) si volge verso i due poeti che sono intenti a conversare tra loro, come lascia intendere il gesto di Stazio: egli sta indicando qualcosa a Virgilio, forse un dettaglio di quel Paradiso Terrestre che sognarono componendo i loro poemi. Con questo il canto si conclude.

\section{Il Paradiso Terrestre dantesco e la Primavera DI BOTTICELLI}

È proprio nell'incontro tra Dante e Matelda che traspare il profondo interesse di Botticelli per Dante, e più precisamente per il Paradiso Terrestre descritto dal poeta, che emerge in modo evidente anche in altre opere del pittore. Nel I882, subito dopo che i disegni botticelliani vennero acquistati per i musei berlinesi, il direttore del Kupferstichkabinett Friedrich Lippmann scrisse 
riguardo a questi fogli: "Jene Scene, wo Dante am Eingang in den Hain des Purgatorio das Blumen pflückende und singende Weib erblickt (Purg. XXVIII) steht an entzückender Lieblichkeit ebenbürtig neben dem 'Frühling' der florentiner Akademie" (Lippmann 1883: 66).

Infatti, la celebre Primavera di Botticelli [fig. 8], conservata presso la Galleria degli Uffizi a Firenze, databile in un momento leggermente precedente rispetto al disegno del canto XXVIII del Purgatorio (I482 ca.), ha un "inescapably poetic character...which in its imagery and style is so evocative of the rich pastoral tradition in Western literature" (Marmor 2003: 199). Il dipinto rappresenta l'ambiente del Paradiso Terrestre dantesco in modo esemplare, tanto che Max Marmor - come numerosi altri studiosi (cfr. Barolsky I983; cfr. Bashir-Hecht 1985) - in uno studio dettagliato sull' argomento ha sottolineato quale sia il legame speciale della tavola con il ciclo di disegni danteschi del Botticelli: "Scarcely anyone who has studied Botticelli's surviving illustrations in Berlin and Rome has failed to note the kinship in style, imagery, and sensibility between his evocative renderings of the Earthly Paradise [...] as described in the final cantos of the Purgatorio, and the Primavera" (Marmor 2003: 203).

La bibliografia relativa a questo capolavoro della pittura del Rinascimento italiano è vastissima e le interpretazioni dell'opera si estendono in ogni direzione immaginabile. Mi limiterò qui ad approfondire solo alcuni parallelismi tra il dipinto e il disegno del canto ventottesimo del Purgatorio. Infatti, la Primavera introduce altri episodi rispetto al disegno e con essi ulteriori livelli di significato derivanti da alcuni passaggi del canto. Tra la descrizione di Dante e la rappresentazione di Botticelli esiste un'affinità stringente per quanto riguarda lo stile e le immagini. Questo vale soprattutto per la figura di Matelda, la quale già nelle parole di Dante è delineata come un personaggio di straordinaria complessità (cfr. Marmor 2003: 204; cfr. Grimaldi Pizzorno I994; cfr. Bashir-Hecht 1985: I033): essa infatti rievoca le figure mitologiche di Flora e Proserpina mentre sta "cantando e scegliendo fior da fiore / ond'era pinta tutta la sua via" ( $P g$. XXVIII, 4I-42): "Tu mi fai remembrar dove e qual era / Proserpina nel tempo che perdette / la madre lei ed ella primavera" ( $P g$. XXVIII, 49-5I). In questo Matelda è molto simile alla figura con la veste a fiori, generalmente identificata come Flora o Proserpina, che sta a destra nel dipinto di Botticelli. Anche la donna al centro del quadro richiama la descrizione che Dante fa di Matelda nel canto ventottesimo del Purgatorio, in quanto tutte e due sono custodi di un luogo naturale di divina bellezza. Mentre in Dante il paragone tra Matelda e la Madonna è poco più di un'allusione velata ("non altrimenti / che vergine che li occhi onesti avvalli" - Pg. XXVIII, 56-57), quella alla dea dell'amore è esplicito ("Non credo che splendesse tanto lume / sotto le ciglia a Venere, trafitta /dal figlio fuor di 
tutto suo costume. / Ella ridea da l'altra riva dritta, / trattando più color con le sue mani, / che l'alta terra sanza seme gitta" - Pg. XXVIII, 64-69). Tanti studiosi, tra l'altro anche Ernst Gombrich, riconobbero che entrambe le allusioni, cioè alla Madonna e a Venere, erano presenti nella figura centrale del dipinto (cfr. Gombrich 1945: 4I). Inoltre, in tale figura - che incarna l'intero significato del quadro ovvero la Primavera - si può riconoscere un terzo personaggio femminile: Matelda. Infatti, sia nella Matelda descritta da Dante che in quella disegnata da Botticelli nel centro del foglio del nostro canto si possono individuare vari riferimenti formali alle figure femminili rispettivamente a destra ed al centro del dipinto: la veste dai panni fluttuanti ed il passo quasi danzante ricordano Flora o Proserpina, il cui gesto di raccogliere la veste al ventre è una declinazione più marcata dello stesso gesto compiuto sia dalla Primavera che da Matelda. I personaggi del dipinto che dal punto di vista formale sono più affini alla Matelda del disegno sono una delle Grazie (e in questo tematicamente anche una figura affine a Venere) e la figura di Mercurio, raffigurato sulla sinistra del dipinto nell'atto di cacciare le nuvole con il caduceo. Proprio questo gesto di Mercurio è riecheggiato dalle parole di Matelda, che rispondendo alle domande di Dante sul Paradiso Terrestre tenta di scacciare le nuvole e la nebbia - metafora del dubbio e delle domande - che appannano la mente del poeta: "ma luce rende il salmo Delectasti, che puote disnebbiar vostro intelletto"; "Io dicerò come procede / per sua cagion ciò ch'ammirar ti face, / e purgherò la nebbia che ti fiede." (Pg. XXVIII, 8I; 88-90) (cfr. Marmor 2003: 207).

Abbiamo detto che l'incontro tra Dante e Matelda costituisce una prefigurazione degli incontri tra Dante e Beatrice nel Paradiso, anche dal punto di vista formale, dal momento che mimica e posizione delle due donne sono molto simili nei vari disegni. Matelda è una prefigurazione di Beatrice, quella che la annuncia, che viene prima di lei; allo stesso modo, come spiega Dante nella Vita Nuova, la "Primavera", verrà prima: “... io vidi venire verso me una gentile donna, la quale era di famosa bieltade ... E lo nome di questa donna era Giovanna, salvo che per la sua bieltade, secondo che altri crede, imposto l'era nome Primavera; e così era chiamata. E appresso lei, guardando, vidi venire la mirabile Beatrice. Queste donne andaro presso di me così l'una appresso l'altra, e parve che Amore mi parlasse nel cuore, e dicesse: 'Quella prima è nominata Primavera solo per questa venuta d'oggi; ché io mossi lo imponitore del nome a chiamarla così Primavera, cioè prima verrà lo die che Beatrice si mostrerà dopo la imaginazione del suo fedele"' (Vita Nuova XXIV,4; cfr. Grimaldi Pizzorno 1994: I23-I24).

Tutto questo contribuisce alla fitta rete di riferimenti tra rappresentazione illustrativa e contenuto poetico del quadro, una sceneggiatura che corrisponde intrinsecamente a quella del Paradiso terrestre. In questo senso il dipinto 
offre - oltre a tanti altri riferimenti e motivi - una sintesi visiva della Matelda dantesca, che viene messa a fuoco ed analizzata nei suoi molteplici significati. Tuttavia, se nella pittura su tavola Botticelli introduce anche elementi non legati al mondo dantesco (cfr. Zöllner 1997), nel caso del disegno - arte che più di ogni altra avvicina l'occhio dello spettatore alla mano dell'artista - egli si attiene con scrupolosa attenzione ai concetti e agli avvenimenti descritti dal poeta. La linea in movimento evoca allo stesso tempo sia il viaggio interiore che quello esteriore compiuto da Dante, disegnando e visualizzando gli spazi che il poeta vede e misura a grandi passi. Da questo punto di vista, Botticelli non solo commenta Dante, ma lo accompagna da vicino, marcandone le tracce con la penna del disegnatore. Ed è proprio "nelle mistiche visioni del Paradiso Terrestre che l'arte del Botticelli dimostra le sue qualità supreme" (Toesca 1922: 15). Botticelli, che secondo Vasari non possedeva alcuna erudizione, in questi disegni crea un commento illustrato dell'universo dantesco, interpretandolo e modellandolo attraverso il linguaggio dell'arte e si manifesta così oltre pictor doctus anche delineator doctus per eccellenza. 


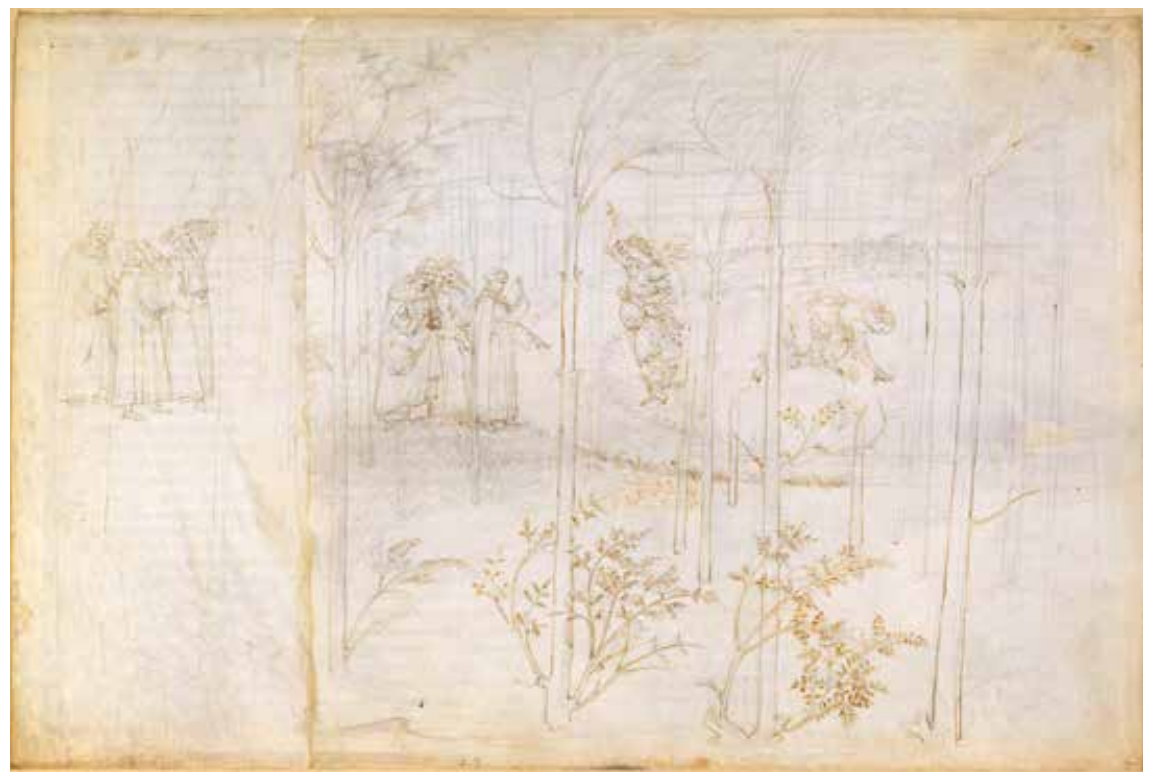

Fig. I. Sandro Botticelli, Il Paradiso Terrestre: Purgatorio XXVIII, I488-I492 ca., penna e inchiostro marrone, punta metallica su pergamena, Berlino, Staatliche Museen zu Berlin Kupferstichkabinett.

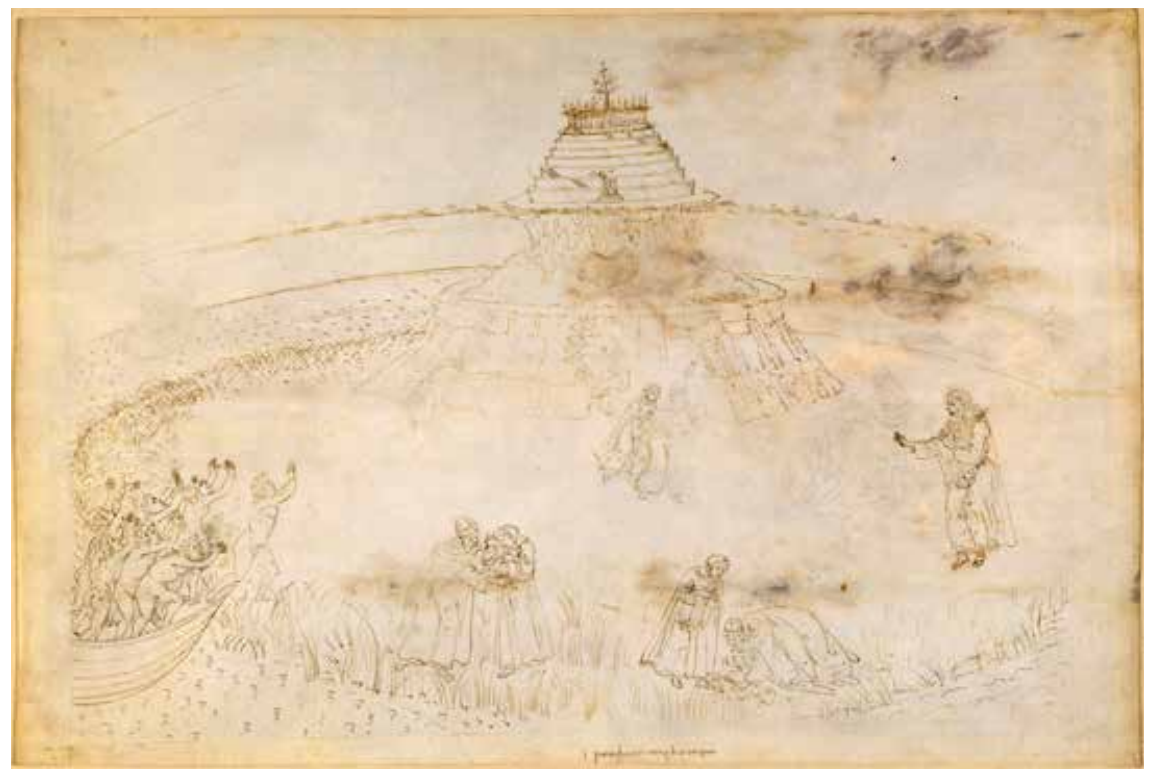

Fig. 2. Sandro Botticelli, La montagna del Purgatorio: Purgatorio I, I488-I492 ca., penna e inchiostro marrone e punta metallica su pergamena, Berlino, Staatliche Museen zu Berlin Kupferstichkabinett. 


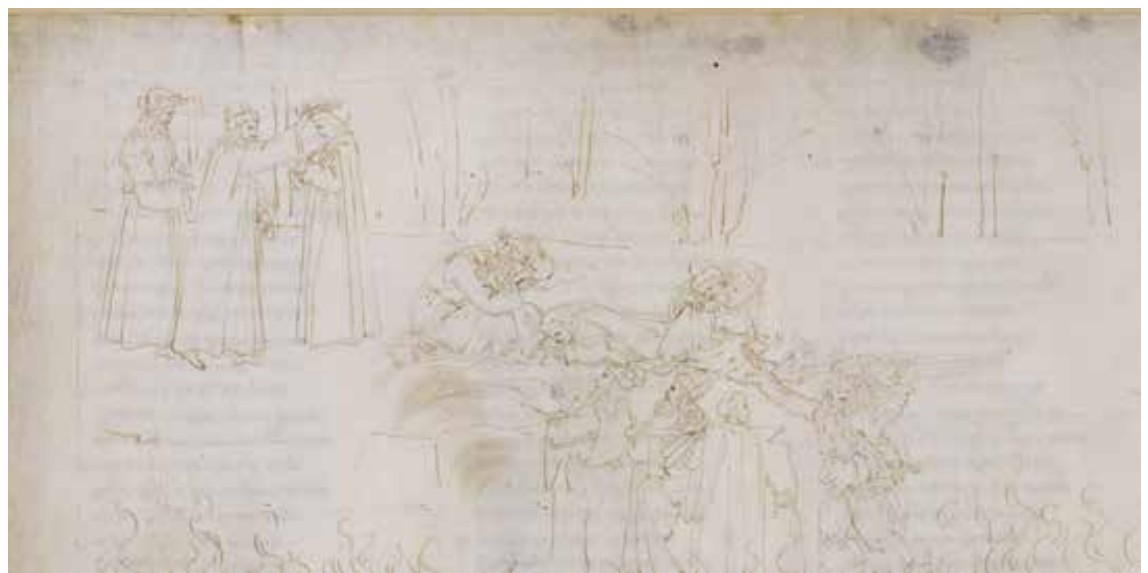

Fig. 3. Sandro Botticelli, Ascesa al Paradiso Terrestre: Purgatorio XXVII (dettaglio), I488-I492 ca., penna e inchiostro marrone e punta metallica su pergamena, Berlino, Staatliche Museen zu Berlin - Kupferstichkabinett.

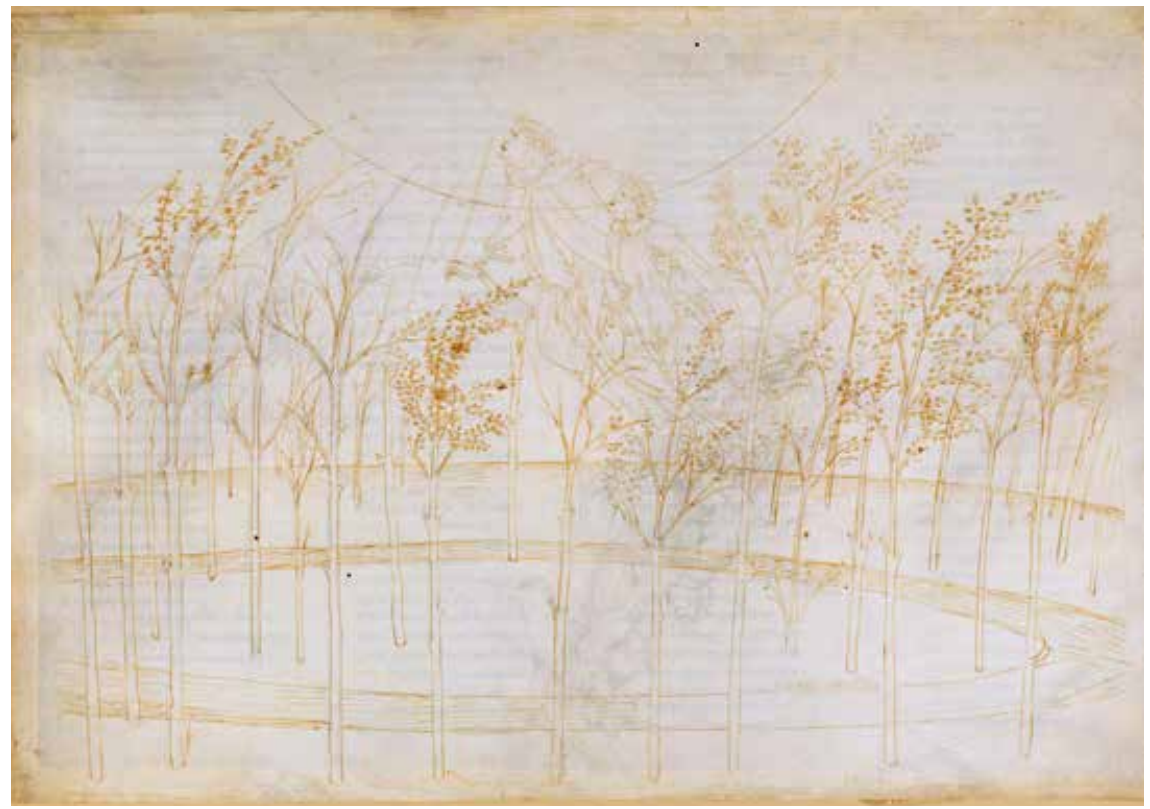

Fig. 4. Sandro Botticelli, Ascesa alla sfera del fuoco: Paradiso I, I492 ca., penna e inchiostro marrone e punta metallica su pergamena, Berlino, Staatliche Museen zu Berlin - Kupferstichkabinett. 


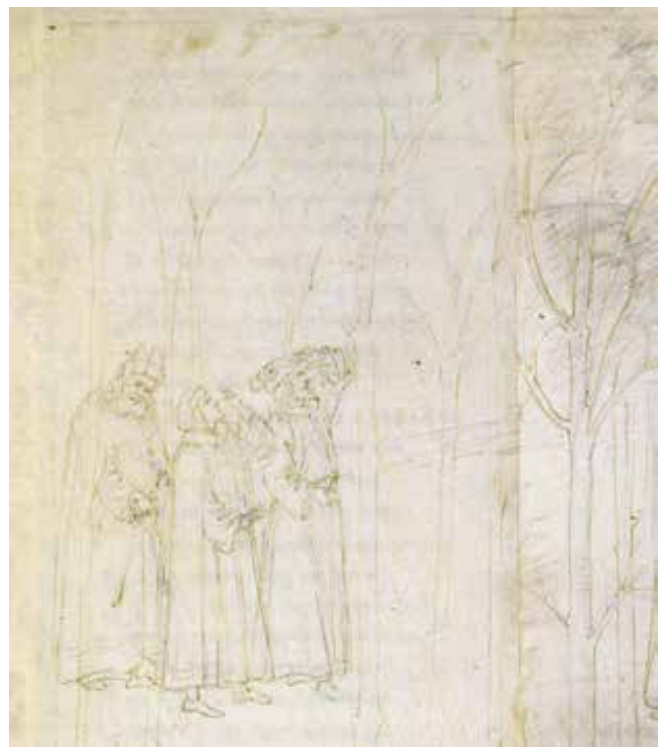

Fig. 5. Sandro Botticelli, Il Paradiso Terrestre: Purgatorio XXVIII (dettaglio), I488-I492 ca., penna e inchiostro marrone e punta metallica su pergamena, Berlino, Staatliche Museen zu Berlin - Kupferstichkabinett.

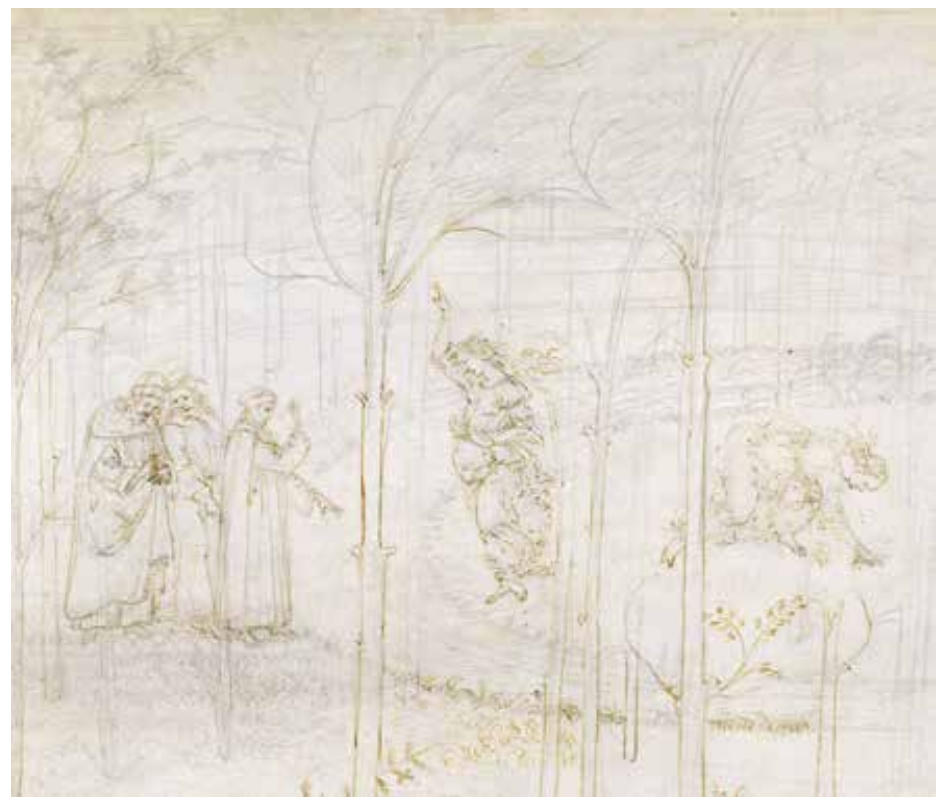

Fig. 6. Sandro Botticelli, Il Paradiso Terrestre: Purgatorio XXVIII (dettaglio), I488-I492 ca., penna e inchiostro marrone e punta metallica su pergamena, Berlino, Staatliche Museen zu Berlin - Kupferstichkabinett. 


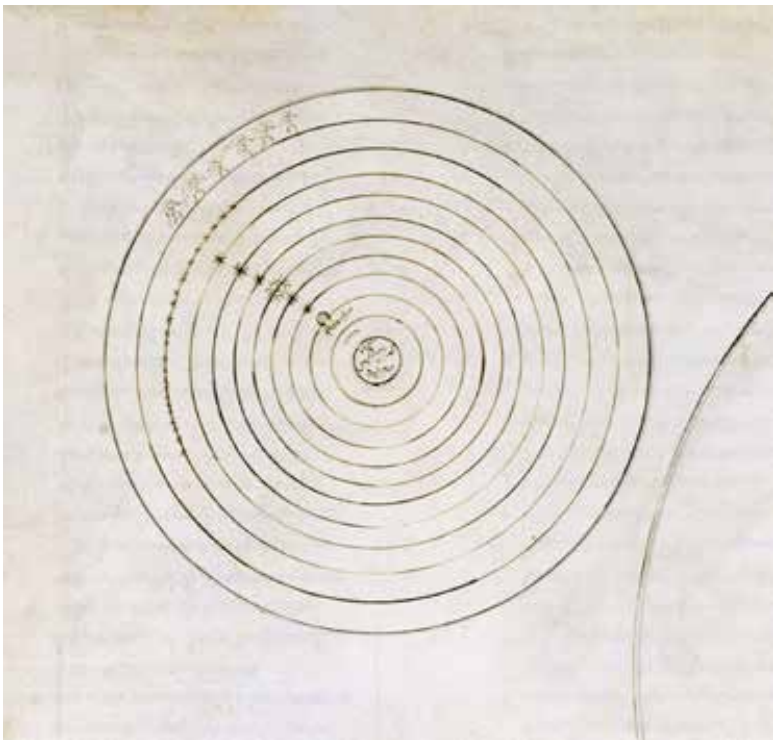

Fig. 7. Sandro Botticelli, Il sistema delle sfere celesti: Paradiso II (dettaglio), I492 ca., penna e inchiostro marrone e punta metallica su pergamena, Berlino, Staatliche Museen zu Berlin Kupferstichkabinett.

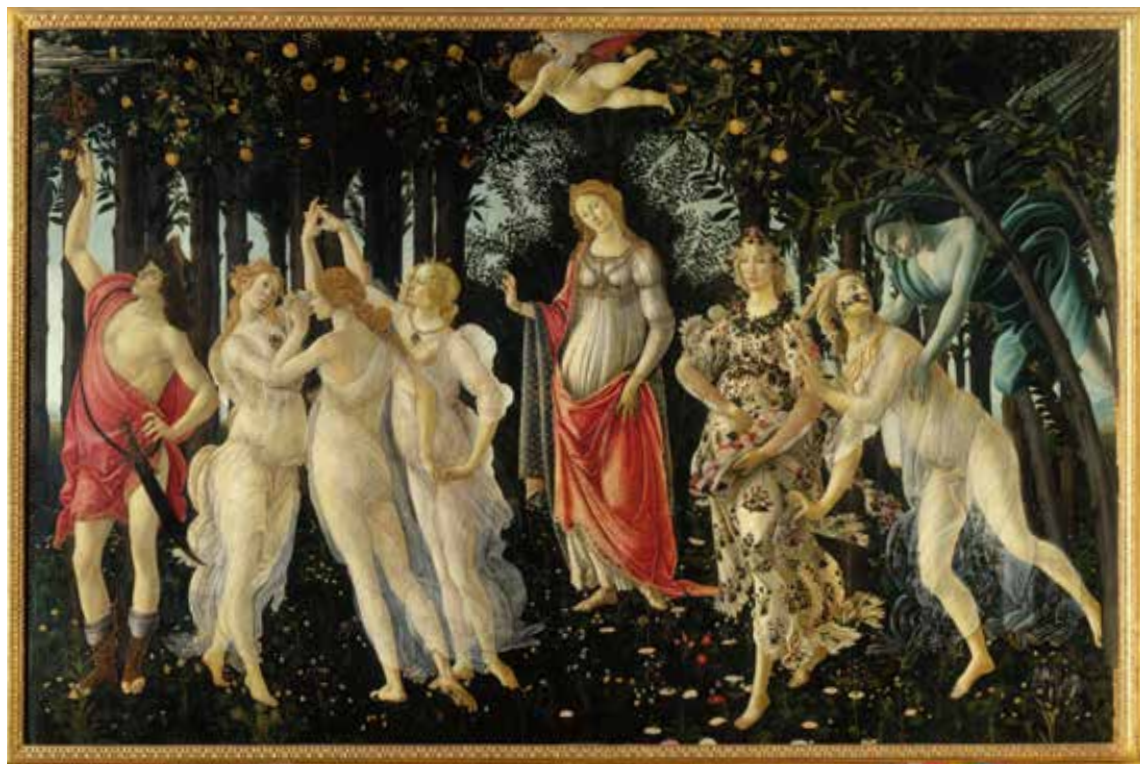

Fig. 8. Sandro Botticelli, Primavera, I 482 ca., dipinto su tavola, Firenze, Galleria degli Uffizi. 


\section{BibLIOGRAFIA:}

\section{Fonti principali:}

Alighieri, Dante, La Commedia, a cura di Giorgio Petrocchi, Milano, Mondadori, I966.

Alighieri, Dante, La Vita Nuova di Dante Alighieri, a cura di Michele Barbi, Firenze, Bemporad \& Figlio, 1932.

Vasari, Giorgio, Le vite de' più eccellenti pittori, scultori e architettori: nelle redazioni del I550 e I568, cura di Rosanna Bettarini e di Paola Barocchi, Firenze, Sansoni, I987.

\section{Fonti secondarie:}

Bashir-Hecht, H., 1985, "Die Paradieslandschaft bei Dante und Botticelli", Die Kunst und das schöne Heim, 97, I03I-I034.

Barolsky, P., I983, "Botticelli's Primavera and the tradition of Dante", Konsthistorisk tidskrift, 52, I-6.

Batard, Y., 1952, Les dessins de Sandro Botticelli pour la Divine Comédie, Paris, Perrin (Collection Jeu savant, 2).

Bellini P., 1990, "Le due serie di disegni del Botticelli per la Commedia", Gizzi C. (a cura di), Botticelli e Dante, Milano, Electa, 4I-50.

Clark, K., 1976, The Drawings by Sandro Botticelli for Dante's Divine Commedy after the Originals in the Berlin Museums and the Vatican, London, Thames \& Hudson.

Dombrowski, D., I992, Die Divina Commedia als Stätte der Ganzheit. Sandro Botticellis Dante-Visionen, Westfälische Wilhelms-Universität Münster (tesi di laurea/Magister-Arbeit non pubblicata).

Dombrowski, D., I996, "Beobachtungen zu Botticellis Dante-Illustrationen", Deutsches Dante-Jahrbuch, 7I, 45-75.

Dreyer, P., 1984, "Botticelli's series of Engravings of I48I", Print Quarterly, I, 2, III-II5.

Fallani, G., I97I, Dante e la cultura figurativa medievale, Bergamo, Minerva Italica.

Gentile, S. (a cura di), 200o, Sandro Botticelli. Pittore della Divina Commedia, vol. I, Milano, Skira.

Girolami Cheney, L. de, I988, "I disegni di Botticelli per la Divina Commedia di Dante", Letteratura italiana e arti figurative (Franceschetti, A., a cura di), vol. I. Firenze, Leo S. Olschki, 26I-267.

Gizzi, C. (a cura di), I990, Botticelli e Dante, Milano, Electa.

Gombrich, E. H., 1945, "Botticelli's Mythologies: A Study in the Neoplatonic Symbolism of His Circle", Journal of the Warburg and Courtauld Institutes, 8, 7-6o.

Grimaldi Pizzorno, P., 1994, "Matelda's Dance and the Smile of the Poets", Dante Studies, with the Annual Report of the Dante Society, II2, II5-I32.

Keller, P., 200o, "Die Kupferstiche zur Ausgabe der Göttlichen Komödie von I48I", Schulze Altcappenberg, H.-Th. (a cura di), Sandro Botticelli. Der Bilderzyklus zu Dantes Göttlicher Komödie. Mit einer repräsentativen Auswahl von Zeichnungen Botticellis und illuminierten Commedia-Handschriften der Renaissance, OstfildernRuit/London, Hatje-Cantz - Royal Academy of Arts, 326-333.

Klettke, C., 20Io, "Paradiesmystik im Grenzbereich des Nicht-Darstellbaren. Par. XXX: Dantes Wortgemälde und Botticellis Zeichnung. Analyse eines 
Medienwechsels", Letteratura\& Arte, 8 (= Mattusch, M. e Setzkorn, S., a cura di, Immaginazione-Evocazione-Immagine. Dimensioni Teorico-Estetiche del rapporto Testo-Immagine nella Letteratura Italiana. Atti del Convegno di Berlino (Humboldt-Universität zu Berlin, 8-Io maggio 2008), Pisa/Roma, Fabrizio Serra, $55-83$.

Lightbown, R., 1978, Sandro Botticelli. Complete Catalogue, London, Paul Elek.

Lippmann, F., I883, "Die Zeichnungen des Sandro Botticelli zu Göttlichen Komödie", Jahrbuch der Preußischen Kunstsammlungen, 4, I883, 63-72.

Lippmann, F., $192 \mathrm{I}^{2}$, Zeichnungen von Sandro Botticelli zu Dantes Göttlicher Komödie. Verkleinerte Nachbildungen der Originale im Kupferstichkabinett zu Berlin und in der Bibliothek des Vatikans, mit einer Einleitung und der Erklärung der Darstellungen, Berlin, Grote.

Marmor, M. C., 2003, "From Purgatory to the "Primavera": Some Observations on Botticelli and Dante", in: Artibus et Historiae" 24, No. 48, I99-2I2.

Oltrogge, D. / Fuchs, R. / Hahn, O., 200o, "Finito und Nonfinito. Zur Technik von Zeichnung und Malerei in Botticellis Divina Commedia", Schulze Altcappenberg, H.-Th. (a cura di), Sandro Botticelli. Der Bilderzyklus zu Dantes Göttlicher Komödie. Mit einer repräsentativen Auswahl von Zeichnungen Botticellis und illuminierten Commedia-Handschriften der Renaissance, Ostfildern-Ruit/ London, Hatje-Cantz - Royal Academy of Arts, 334-34I.

Risset, J., 2008, "De Botticelli à Dante. Naissance de Beatrice”, Selliers, D. de (a cura di), La Divine Comédie. Dante Alighieri. Illustrations. Sandro Botticelli, Firenze, Le Lettere, II-25.

Schulze Altcappenberg, H.-Th., 20oob, “" "per essere persona sofistica”. Il ciclo botticelliano per la Divina Commedia”, in: Schulze Altcappenberg, H.-Th. (a cura di), Sandro Botticelli. Pittore della Divina Commedia, Vol. 2, Milano, Skira, 13-35.

Schulze Altcappenberg, H.-Th. (a cura di), 2000a, Sandro Botticelli. Der Bilderzyklus zu Dantes Göttlicher Komödie. Mit einer repräsentativen Auswahl von Zeichnungen Botticellis und illuminierten Commedia-Handschriften der Renaissance, OstfildernRuit/London, Hatje-Cantz - Royal Academy of Arts.

Stapleford, R., 1995, "Vasari and Botticelli", Mitteilungen des Kunsthistorischen Institutes in Florenz, 39, 2/3, 397-408.

Toesca, P., I922, "Sandro Botticelli e Dante", La Bibliofilia. Rivista di Storia del libro $e$ delle arti grafiche, XXIV, I-I9.

Venturi, A., I92I, Il Botticelli interprete di Dante, Firenze, Le Monnier.

Warburg, A., I893, Sandro Botticelli's "Geburt der Venus" und "Frühling". Eine Untersuchung über die Vorstellungen von der Antike in der italienischen Frührenaissance, Hamburg/Leipzig, Verlag von Leopold Voss.

Zöllner, F., I997, "Zu den Quellen und zur Ikonographie von Sandro Botticellis Primavera", Wiener Jahrbuch für Kunstgeschichte, 50, I3I-I58; 357-366.

\section{Copyright foto:}

Figg. I-7: (C) Staatliche Museen zu Berlin - Kupferstichkabinett / Philipp Allard Fig. 8: (C) Photo Scala, Florence - courtesy of the Ministero Beni e Att. Culturali 